\title{
Lower endogenous p53 levels and degradation of AKT protein contribute to potent suppression of the new antibiotic Xiakemycin A on tumor cells
}

\author{
CHUAN CHEN $^{1,2}$, ZHONGKE JIANG ${ }^{1}$, XIUYUAN OU ${ }^{1}$, HUIXIAN ZHANG ${ }^{1}$, \\ CHENGHANG SUN $^{1}$ and QIYANG HE ${ }^{1}$ \\ ${ }^{1}$ Institute of Medicinal Biotechnology, Chinese Academy of Medical Sciences and Peking Union Medical College, \\ Beijing 100050; ${ }^{2}$ Faculty of Basic Medical Sciences, Jiujiang University, Jiujiang, Jiangxi 332000, P.R. China
}

Received July 27, 2017; Accepted March 28, 2018

DOI: $10.3892 / o r .2018 .6380$

\begin{abstract}
Xiakemycin A (XKA), a new pyranonaphthoquinone antibiotic, is isolated from the fermentation broth of Streptomyces sp. CC8-201. It exerts potent suppression of cell proliferation on some types of tumor cells. In the present study, its underlying mechanism on tumor cells has been investigated. In contrast to the specific AKT inhibitor triciribine hydrate, XKA demonstrated a weak inhibition of the AKT kinase activity in vitro. Knockdown of AKT protein levels reduced XKA-inhibitory action on prostate carcinoma PC-3 cells. Degradation of AKT protein was markedly observed in the XKA-treated PC-3 cells in comparison with triciribine hydrate treatment. There was no typical apoptosis induced by XKA in PC-3 cells. The propidium iodide-stained cells increased concentration-dependently when the cells were treated with XKA. Degradation of apoptosis-related proteins, such as p53 and PARP-1, was also detected in the XKA-treated PC-3 cells. Knockdown of p53 protein levels potentiated XKA action on non-small lung cancer A549 cells. Collectively, the mechanism of XKA potent inhibition was due to degradation of AKT protein and low endogenous p53 levels. As a leading compound, new derivatives based on XKA will be developed to precisely treat tumor cells which have high AKT and low p53 protein levels.
\end{abstract}

\section{Introduction}

AKT kinase, also known as protein kinase $\mathrm{B}$, is an important regulator for several growth factors and signaling pathways,

Correspondence to: Dr Chenghang Sun or Dr Qiyang He, Institute of Medicinal Biotechnology, Chinese Academy of Medical Sciences and Peking Union Medical College, 1 Tiantan Xili, Beijing 100050, P.R. China

E-mail: chenghangsun@hotmail.com

E-mail: qiyang_he@vip.163.com

Key words: Xiakemycin A, AKT kinase inhibitor, antitumor antibiotic, p53 protein, pyranonaphthoquinone and activates a series of downstream substrates involving cell proliferation, cell survival, stress response and metabolism (1). Molecular elucidation of the AKT signaling pathways and epidemiological investigations have firmly established a critical role for AKT in human tumorigenesis and chemoresistance (2-4). Therefore, AKT is a potential target for developing new antitumor agents. Four types of AKT specific inhibitors have been developed, which include the compounds that target the ATP binding pocket, allosteric inhibitors, inhibitors targeting the PH domain or pseudosubstrate inhibitors. However, due to poor selectivity, toxicity, poor solubility, unacceptable pharmacokinetics or inhibitory inactivition in phase II, none of the AKT inhibitors has been approved for clinical application up to date $(2,5,6)$.

Xiakemycin A (XKA) is a new member of the pyranonaphthoquinone (PNQ) family of antibiotics. As the first Chinese karst cave-originated new antibiotic, XKA is isolated from the fermentation broth of Streptomyces sp. CC8-201 (7). PNQ antibiotics have the basic skeleton of naphtho[2,3-c]pyran-5, 10-dione ring system and display a range of interesting biological activities, including inhibitory activities against tumor, fungi, bacteria, insect, virus and mycoplasma $(8,9)$. Concerning antitumor action, some PNQ compounds were revealed to target several important molecules, such as AKT, protein kinase A and DNA topoisomerase II (10-12). However, the mechanisms underlying the signaling pathway and tumor cell deaths induced by any of the PNQ antibiotics remain unclear.

In the present study, tumor cell death induced by XKA and its action on AKT and p53 were investigated. The results firstly showed that XKA degraded cellular AKT protein, and that cellular endogenous p53 protein levels were among the determining factors for the action of XKA.

\section{Materials and methods}

Drugs and chemicals. XKA at $>95 \%$ purity was isolated from the fermentation broth of Streptomyces sp. CC8-201 and purified by the previously described method (7). It was prepared into a $5 \mathrm{mM}$ solution with methanol and stored at $-20^{\circ} \mathrm{C}$ before use. Dimethyl sulfoxide (DMSO),3-(4,5-dimethyl-2-thiazolyl)2,5-diphenyl-2H-tetrazolium bromide (MTT), triciribine 
hydrate (TCN) and propidium iodide (PI) were all purchased from Sigma-Aldrich (Merck KGaA, Darmstadt, Germany). In addition, 2,7-dichlorfluorescin diacetate ( $\left.\mathrm{H}_{2} \mathrm{DCFDA}\right)$, RPMI-1640 and Dulbecco's modified Eagle's medium (DMEM) were obtained from Invitrogen (Thermo Fisher Scientific, Inc., Waltham, MA, USA ).

Cell lines and cultures. Human hepatoblastoma HepG2, non-small lung cancer A549, breast cancer MCF-7, prostate carcinoma PC-3, colon carcinoma HCT 116, cervical cancer HeLa and human neuroblastoma SH-SY5Y cell lines were all purchased from Shanghai Cell Bank, Institute of Biochemistry and Cell Biology, Chinese Academy of Sciences. The STR profiles of all the cell lines were confirmed by the provider. Concerning the HepG2 cell line it is misidentified according to: (https://www.ncbi.nlm.nih.gov/pubmed/19751877). This cell line was thought to be derived from a hepatocellular carcinoma (HCC) tumor but it has since been shown to be from hepatoblastoma. It has not affected the outcomes of this study.

All the cells were cultured in RPMI-1640 or DMEM supplemented with $10 \%$ fetal bovine serum (FBS; Tianjin Haoyang Biotech Co., Tianjin, China). The cells were incubated at $37^{\circ} \mathrm{C}$ with $5 \% \mathrm{CO}_{2}$ in a humidified atmosphere.

MTT assay. Cell viability was assessed by an MTT assay. Cells were seeded in a 96-well plate for $24 \mathrm{~h}$ at a cell density of $3 \times 10^{3} /$ well and then treated with XKA for $72 \mathrm{~h}$. The final concentration of methanol in the culture media was $<0.1 \%$. MTT method was performed as previously described (13). The control group was set as $100 \%$, and $\mathrm{IC}_{50}$ values were assessed using the non-linear regression analysis.

Western blot analysis. Western blot assay was performed as previously described (14). The antibodies against cleaved caspase-3 (1:1,000; cat. no. 9664), caspase-9 (1:1,000; cat. no. 9505), AKT (1:1,000; cat. no. 9272), p-AKT (1:1,000; cat. no. 9271) and PARP-1 (1:1,000; cat. no. 9542) were obtained from Cell Signaling Technology, Inc. (Danvers, MA, USA). The antibodies against p53 (1:2,000; cat. no. sc-126) and $\beta$-actin (1:5,000; cat. no. sc-1616) were obtained from Santa Cruz Biotechnology, Inc. (Santa Cruz, CA, USA).

Detection of apoptotic cells by flow cytometry. The apoptotic cells were stained with Annexin V-FITC/PI apoptosis kit (BD Biosciences, San Jose, CA, USA), following the manufacturer's protocol. The fluorescence intensities were assessed using a BD FACSCalibur flow cytometer (BD Biosciences).

RNA interference. Cells were transfected with Lipofectamine 2000 (Invitrogen; Thermo Fisher Scientific, Inc.) according to the manufacturer's instructions at a concentration of $100 \mathrm{pmol}$ siRNA/gene. The sequences of AKT and p53 siRNA were as follows: AKT, 5'-ACGAGUUUGAGUACCUGAA-3' and p53, 5'-GACTCCAGTGGTAATCTAC-3'.

Assessement of reactive oxygen species (ROS). Following treatment with $2 \mu \mathrm{M}$ XKA for different time-points, the cells were stained with $5 \mathrm{mM} \mathrm{H}$ DFFDA for $1 \mathrm{~h}$ at $37^{\circ} \mathrm{C}$. The cells were harvested, and then flow cytometry was performed with

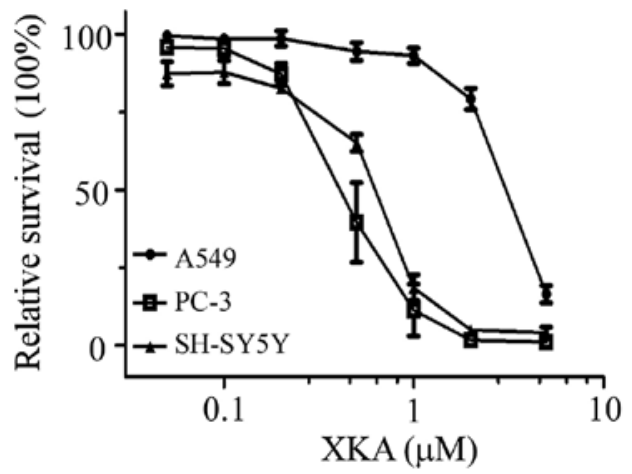

Figure 1. The growth-inhibitory actions of Xiakemycin A (XKA) on PC-3, A549 and SH-SY5Y cells. Cell survival was determined by an MTT assay. The data are the mean \pm SD from three independent experiments.

excitation and emission wavelengths of 488 and $530 \mathrm{~nm}$, respectively.

PI staining. To determine the type of cell death, cells $\left(5 \times 10^{4}\right.$ cells/well) were cultured in 24 -well plates and then treated with XKA. Cells were washed with PBS twice and stained with $50 \mu \mathrm{g} / \mathrm{ml}$ PI for $15 \mathrm{~min}$. The cells were observed and imaged under a fluorescence microscope (Olympus Corporation, Tokyo, Japan).

AKT1 activity assay in vitro. AKT1 assay kit was purchased from BioVision, Inc. (Mountain View, CA, USA). The assay was performed according to the manufacturer's protocol. In brief, the activity of the immunoprecipitated AKT1 was assessed using exogenously recombinant GSK $3 \alpha$ as the substrate. The bands of phosphorylated GSK $3 \alpha$ were determined by western blot analysis.

Statistical analysis. Data are expressed as the means \pm standard deviations (SD). One-way analysis of variance (ANOVA) was used for the inter-group comparison. Statistical analysis was performed using SPSS 17.0 software (SPSS, Inc., Chicago, IL, USA). $\mathrm{P}<0.05$ was considered to indicate a statistically significant result.

\section{Results}

The growth-inhibitory activity of XKA on human tumor cells. In order to assess the cytotoxic activity of XKA, seven human tumor cell lines were used for the MTT assay. As displayed in Fig. 1, the growth-inhibitory activity of XKA on PC-3, SH-SY5Y and A549 cells was concentration-dependent. The $\mathrm{IC}_{50}$ values of XKA on the seven cell lines have been listed in a previous study (7), with a range of 0.43 to $2.77 \mu \mathrm{M}$. The cell line most susceptible to XKA was PC-3.

Inhibition of AKT1 activity by XKA in vitro. In a previous study, it was demonstrated that selective inhibition of AKT kinase activity required the 3,6-dihydro-2 $H$-pyran ring of PNQ (11). XKA possesses this basic structure and possibly exerts an inhibitory activity of AKT. The AKT1 activity assay kit was used to verify this hypothesis. The reaction conditions of the assay kit were optimized to $4 \mathrm{~h}$ for the 
A

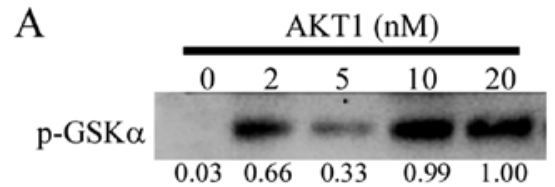

$\mathrm{C}$

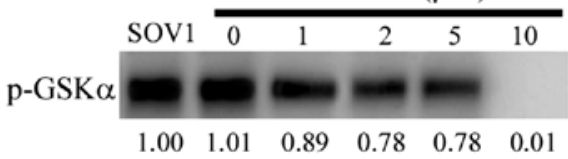

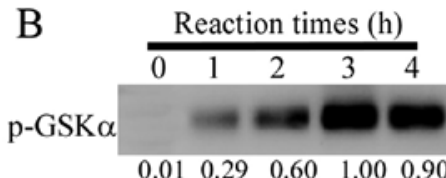

$\mathrm{D}$

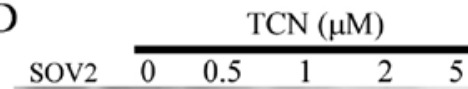
p-GSK $\alpha$

$\begin{array}{llllll}1.00 & 1.02 & 0.82 & 0.71 & 0.42 & 0.01\end{array}$

Figure 2. The effects of Xiakemycin A (XKA) on AKT1 kinase activity in vitro. (A) The proper GSK $\alpha$ activities with different concentrations of AKT1. (B) The proper reaction time for detecting GSK $\alpha$ activities. (C) Effects of XKA on GSK $\alpha$ activities. SOV1, 0.2\% methanol. (D) Effects of TCN on GSK $\alpha$ activities. SOV2, $0.1 \%$ dimethyl sulfoxide (DMSO). TCN, triciribine hydrate.
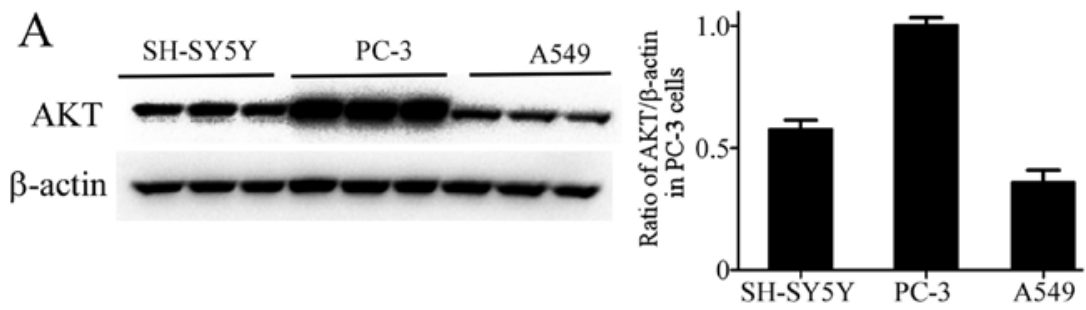

B
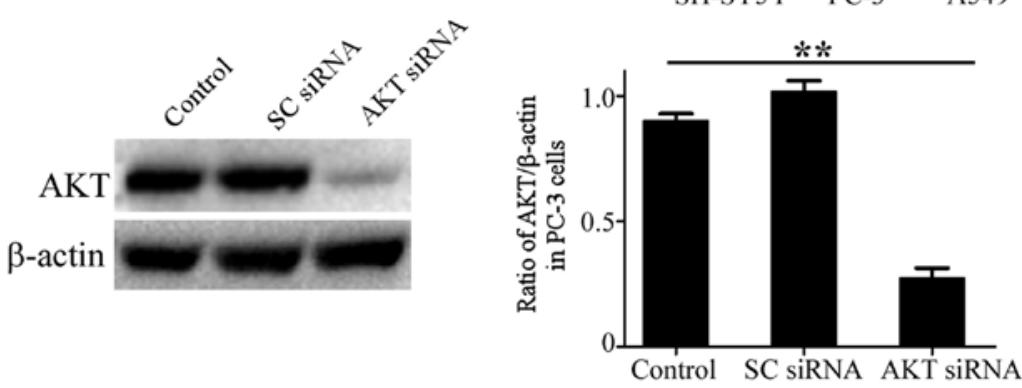

$\mathrm{C}$

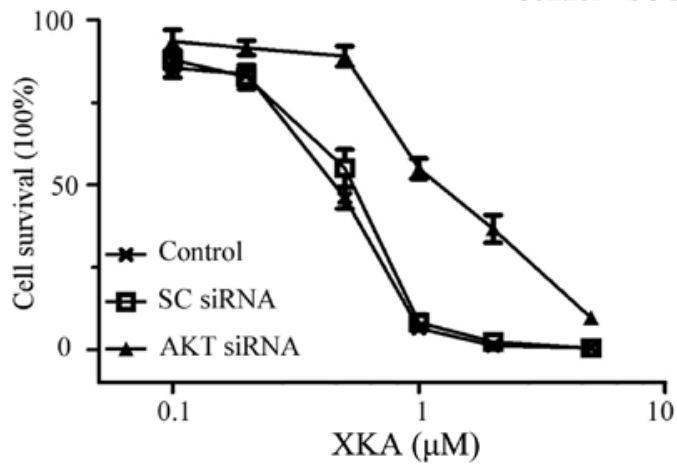

Figure 3. Influence of cellular AKT levels on Xiakemycin A (XKA) action. (A and B) The endogenous AKT levels were determined in various cell lines. ${ }^{* *} \mathrm{P}<0.01$. (C) Knockdown of AKT attenuated the cytotoxicity of XKA on PC-3 cells. Data are expressed as the mean \pm standard deviation from at least three experiments.

reaction time and $10 \mathrm{nM}$ AKT1 for the following determinations (Fig. 2A and B). The suppression of AKT1 activity by TCN was concentration-dependent. In contrast to XKA, there were small changes of the phosphorylated bands from the concentrations of 1 to $5 \mu \mathrm{M}$ (Fig. $2 \mathrm{C}$ ). The $\mathrm{IC}_{50}$ values for inhibiting AKT1 activity by TCN was $1.8 \mu \mathrm{M}$ (Fig. 2D). However, the $\mathrm{IC}_{50}$ values for inhibiting AKT1 activity by XKA was over $5 \mu \mathrm{M}$.

Targeting cellular AKT by XKA. In order to clarify whether AKT is a target of XKA, AKT protein levels from PC-3, SH-SY5Y and A549 cells were detected by western blotting and quantified (Fig. 3A and B). The highest AKT content and potent inhibition of XKA in PC-3 cells indicated that AKT involves XKA action on tumor cells. RNA interference was used to confirm the relationship. AKT protein levels were markedly knocked down in PC-3 cells (Fig. 3C). The XKA susceptibility was significantly reduced and the $\mathrm{IC}_{50}$ value rised to $1.5 \mu \mathrm{M}$, indicating that $\mathrm{AKT}$ is a bona fide target of XKA.

Degradation of AKT protein by XKA in PC-3 cells. In order to decipher the difference between XKA and TCN, AKT phosphorylation was detected by western blotting following 


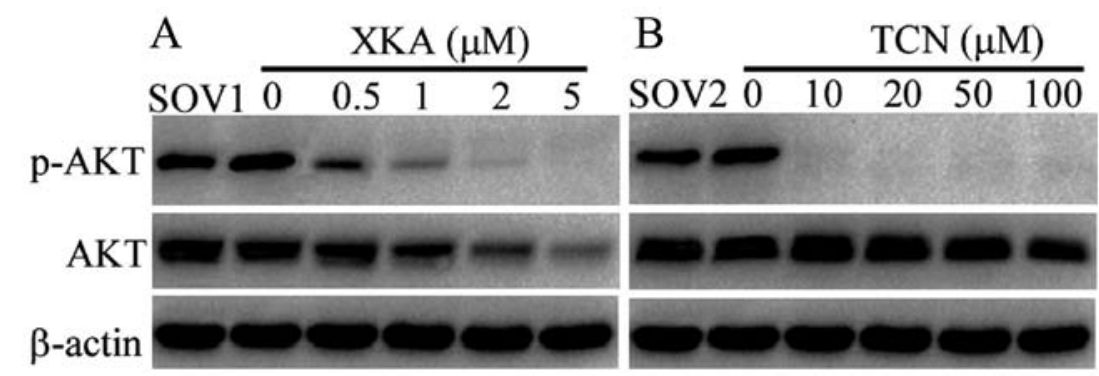

Figure 4. Effects of AKT and p-AKT in PC-3 cells treated with (A) Xiakemycin A (XKA) or (B) triciribine hydrate (TCN). SOV1 in (A), $0.2 \%$ methanol; SOV2 in (B), $0.1 \%$ dimethyl sulfoxide (DMSO). The results are expressed as the mean \pm SD from three separate experiments.
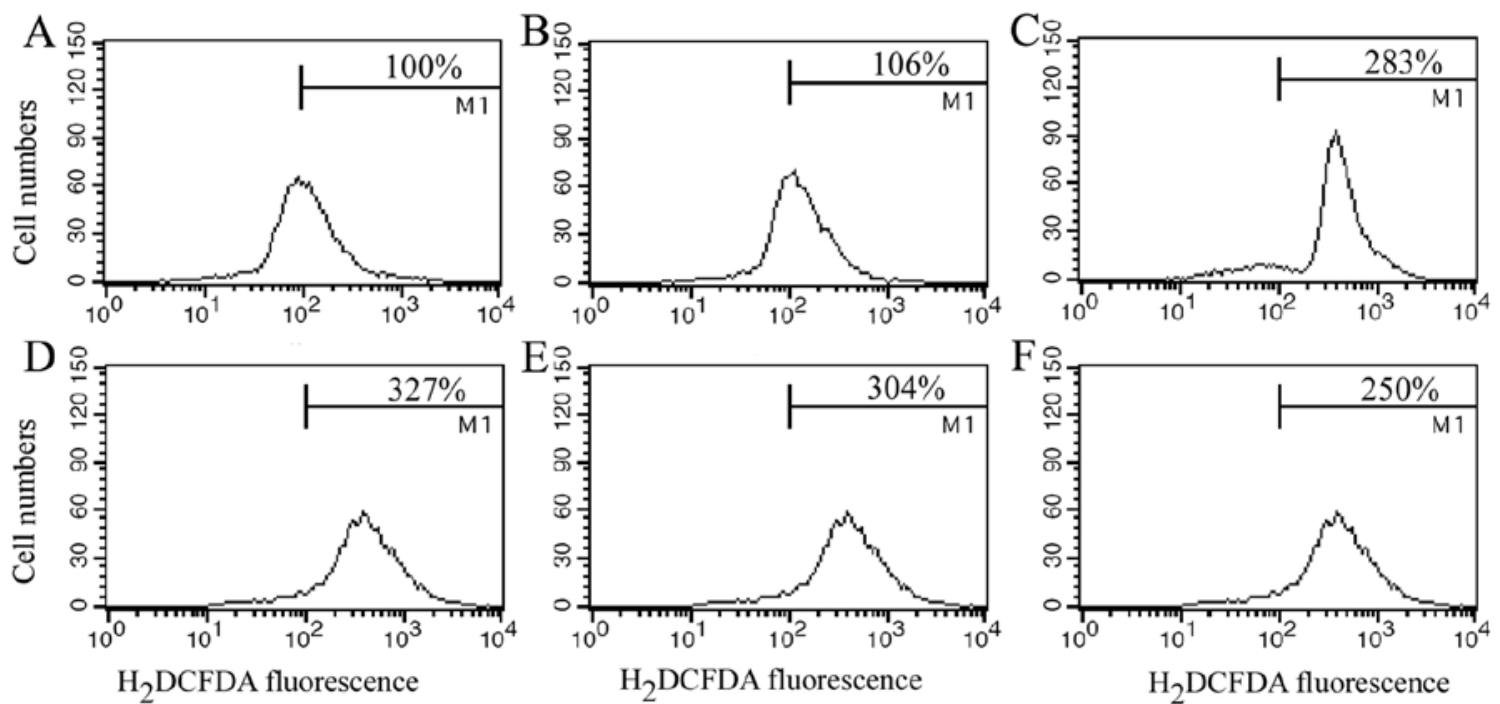

Figure 5. The generation of reactive oxygen species (ROS) triggered by Xiakemycin A (XKA) in PC-3 cells. (A) Control group. (B) Cells treated with $0.2 \%$ methanol. The cells were incubated with $2 \mu \mathrm{M}$ XKA for (C) $1 \mathrm{~h}$, (D) $6 \mathrm{~h}$, (E) $12 \mathrm{~h}$ and (F) $24 \mathrm{~h}$.

exposure to both drugs for $24 \mathrm{~h}$. The phosphorylated AKT disappeared after treatment with $10 \mu \mathrm{M}$ TCN, while the total AKT protein levels remained unchanged when the cells were treated with TCN from 10 to $100 \mu \mathrm{M}$ (Fig. 4). Conversely, $0.5 \mu \mathrm{M}$ XKA treatment significantly reduced phosphorylated AKT levels and the degradation of AKT was obvious following exposure to $1 \mu \mathrm{M}$ XKA. The results demonstrated that suppression of the AKT activity by XKA is related to protein degradation, not kinase inhibition.

Generation of ROS triggered by XKA. In order to explore the mechanism of the potent inhibition of XKA towards tumor cells, ROS was determined with flow cytometry. ROS generation markedly increased after the PC-3 cells were treated with $2 \mu \mathrm{M}$ XKA for $1 \mathrm{~h}$ and higher at $6 \mathrm{~h}$ treatment (Fig. 5), indicating that the XKA treatment triggered oxidant response.

Small ammount of typical apoptotic cells induced by XKA. ROS generation triggered by $2 \mu \mathrm{M}$ XKA verified that the cells were damaged. The ratio of apoptotic cells was detected with Annexin V/PI staining in the XKA-treated PC-3 cells. Approximately $12.4 \%$ apoptotic cells were detected after the PC-3 cells were treated with XKA for $24 \mathrm{~h}$ (Fig. 6A) and it did not increase after exposure to XKA for $48 \mathrm{~h}$, indicating that the majority of cells did not undergo typical apoptosis.
To confirm the Annexin V/PI staining result, PARP-1 and p53 levels were detected by western blotting in PC-3 cells following exposure to $2 \mu \mathrm{M} \mathrm{XKA}$ for $48 \mathrm{~h}$ (Fig. 6B). The cleaved PARP-1 fragment was not observed and degradation of p53 occurred at 6-h treatment. The results further ruled out the possibility that the XKA-induced cell death was apoptosis.

Significant increase of PI-staining cells treated by XKA. In order to identify the type of cell death, PC-3 cells were incubated with $5 \mu \mathrm{M}$ XKA from 1 to $48 \mathrm{~h}$ and PI-stained cells were counted. With the extension of treatment time, the red fluorescent cells increased significantly. At $48 \mathrm{~h}$ of treatment, the cell ratio stained with PI reached 89\% (Fig. 7).

Effect of endogenous p53 levels on XKA action. In order to search other molecules influencing XKA action, p53 protein levels from seven cell lines were detected. The highest level of p53 protein was observed in A549 and HCT-116 cell lines (data not shown), indicating that p53 may be a protein related with the action of XKA.

Knockdown of p53 in the A549 cells was performed and MTT assay was used for determining the susceptibility to XKA. The sensitivity to XKA increased after the reduction of p53 protein levels. The $\mathrm{IC}_{50}$ value decreased to $1.2 \mu \mathrm{M}$ in the p53 siRNA-treated cells (Fig. 8). 


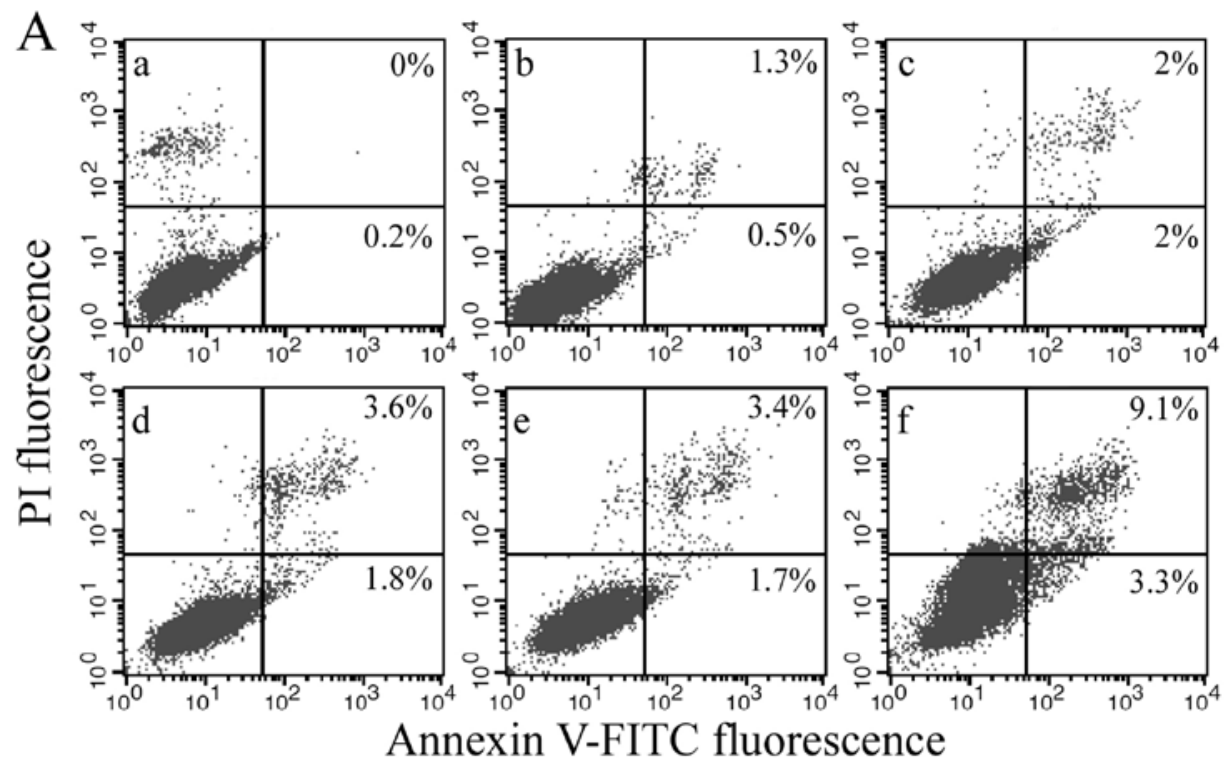

B

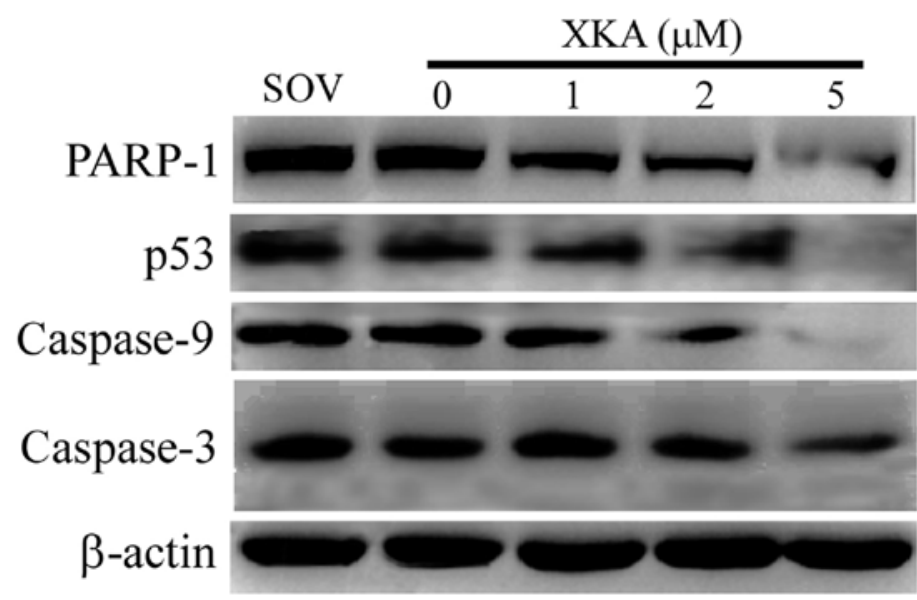

Figure 6. Cell apoptosis induced by Xiakemycin A (XKA). (A) Apoptosis analysis of XKA-treated cells with Annexin V/propidium iodide (PI) staining. (a) Control group; (b) $0.2 \%$ methanol, or treated with $2 \mu \mathrm{M}$ XKA for (c) $1 \mathrm{~h}$, (d) $6 \mathrm{~h}$, (e) $12 \mathrm{~h}$ and (f) $24 \mathrm{~h}$. (B) The levels of apoptosis-related proteins after XKA treatment. A representative result of three independent experiments is displayed.

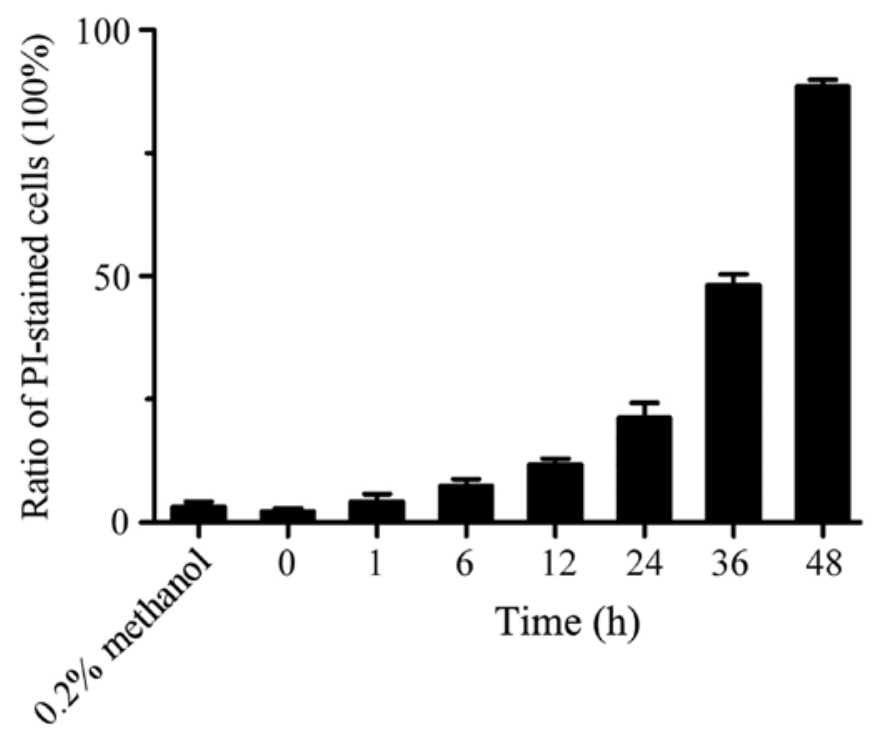

Figure 7. Ratio of propidium iodide (PI)-positive cells following exposure to Xiakemycin A (XKA). The cells were treated with $5 \mu \mathrm{M}$ XKA for $1,6,12$, 24,36 and $48 \mathrm{~h}$ or $0.2 \%$ methanol alone for $48 \mathrm{~h}$. Data are expressed as the mean \pm standard deviation from at least three experiments.

\section{Discussion}

In the present study, we presented evidence that the susceptibility to XKA is positively correlated with the endogenous level of AKT protein and inversely related to that of p53 in tumor cells. The type of cell death induced by XKA may be necroptosis, not apoptosis. To the best of our knowledge, this is the first study to reveal that degradation of AKT was caused by PNQ antibiotics.

PNQ antibiotics are a class of AKT selective kinase inhibitors and the 3,6-dihydro- $2 \mathrm{H}$-pyran ring of the PNQ lactone is an essential structure for potency and selectivity $(10,11)$. Reduction of hydroquinone can subsequently form a quinone methide and this reactive intermediate could then covalently alkylate Cys 310 on the kinase activation, resulting in PNQ lactone-AKT adduct and leading to inhibition of AKT activity. In addition, the T-loop $\mathrm{Cys}^{310}$ residues are critical for sensitivity to the PNQ inhibitors and the T-loop $\mathrm{Cys}^{296}$ residues are indispensable. Through deconstructing analogues to design different steric configuration of basic skeleton and chain structure, researchers have proved that the absolute configuration of 
A
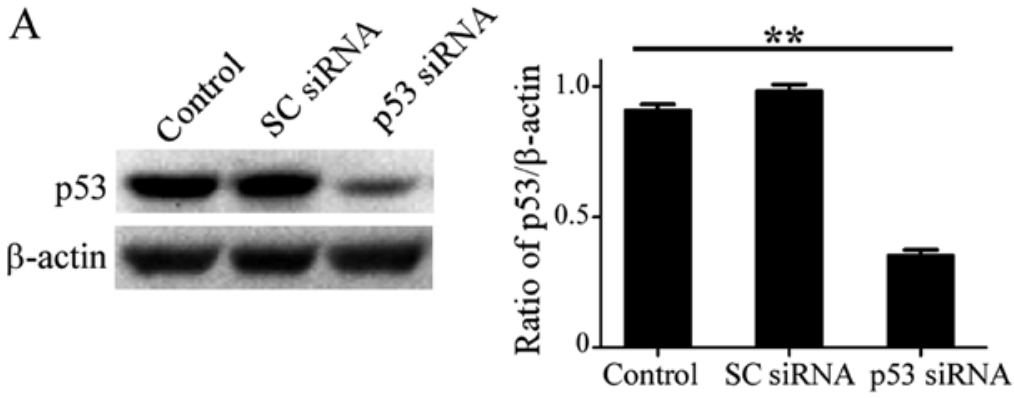

B

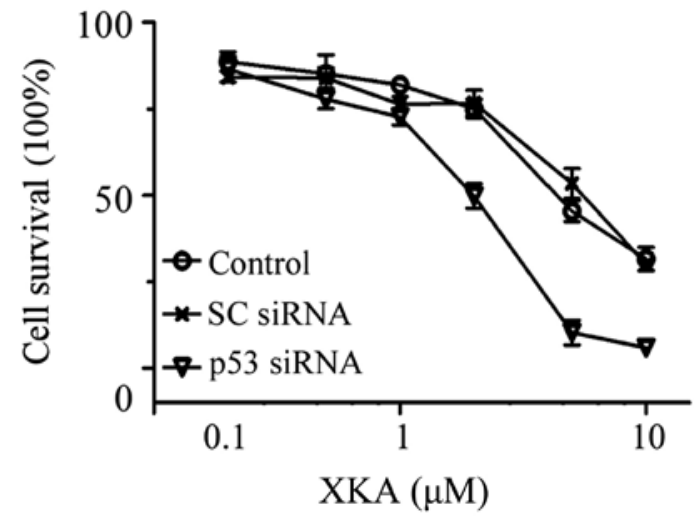

Figure 8. Potentiation of the action of Xiakemycin A (XKA) via knockdown of endogenous p53 levels in A549 cells. (A) Knockdown of p53 levels by siRNA interference. ${ }^{* *} \mathrm{P}<0.01$. (B) Effects of XKA on cell survival after knockdown of p53 levels in A549 cells. The results are representative of two independent experiments.

PNQ affects its inhibitory activity (15). XKA has the essential structural feature for potent and selective inhibition of PNQ, but weak action on inhibiting AKT kinase activity. It is valuable to investigate their structure and AKT inhibition. In the present study, we have only demonstrated AKT degradation in PC-3 cells. Whether AKT is a real target of XKA should be further confirmed in subsequent experiments. The overexpression of AKT protein in A549 or SH-SY5Y cells would clarify this issue.

In the present study, we did not demonstrate why lower AKT protein levels in SH-SY5Y cells have similar susceptibility to XKA as the PC-3 cells. Perhaps there are other molecular factors for the determination of the action of XKA. We aim to further study this difference in our laboratory.

The present study revealed that ROS generation induced by XKA was very rapid due to unique chemical structure. Lactoquinomycin A and XKA have the same basic skeleton (16). The former contains nitrogen in sugar group at $\mathrm{C} 8$, while the latter contains unsaturated sugar at C6. XKA has a higher degree of unsaturation than lactoquinomycin $\mathrm{A}$ and has a high degree of ability as an electron acceptor, leading to generation of superoxide radicals.

According to the results of the present study, reduction of p53 protein levels has enhanced XKA cytotoxicity to tumor cells, indicating one of the biomarkers for predicting the action of XKA. Lower endogenous p53 protein levels had been determined in SH-SY5Y cells (data not shown), which can partially explain its susceptibility to XKA. However, our data are very primary as we did not detect XKA action influenced by the wild-type $\mathrm{p} 53$ or mutant $\mathrm{p} 53$. In further experiments, transfection of HPV E6 protein or addition of $\mathrm{p} 53$ degradation inhibitor may elucidate this issue.
Tumor suppressor protein p53 and AKT kinase play important roles in pro-apoptotic and anti-apoptotic signaling pathways, and determine cellular survival. Accumulating evidence indicates crosstalk between these pathways (17). Ubiquitin ligase Mdm2, the critical p53-regulated enzyme, can be phosphorylated by AKT. Conversely, the activated p53 can also act on AKT in several ways such as caspase-mediated cleavage and self-degradation of AKT, induction of PTEN expression and dephosphorylation of PI3K to suppress AKT activity (18). It has been reported that PTEN deficiency is a biomarker of good response to AKT inhibitor MK-2206 (19). Based on the interaction between p53,PTEN and AKT, rational combination of biomarkers could be promising for predicting AKT inhibitors in different types of tumor cells.

In the present study, the cell death induced by XKA did not present typical apoptosis as the PI-stained cells increased in a concentration-dependent manner. It had been partly suppressed by specific necroptotic inhibitor (data not shown). Therefore, the type of cell death induced by XKA may be necroptosis, which has important roles in physiology as well as diseases (20). The exploration of the underlying mechanism of XKA-induced cell death has been undertaken in our laboratory.

Notably, XKA can degrade the cellular AKT and PARP-1 proteins, indicating a new mode of PNQ antibiotic action on tumor cells. Suppression of the proteasome pathway is an important target of drugs such as bortezomib (21). It is valuable to study the underlying mechanism of protein degradation by XKA.

In conclusion, the underlying mechanism of the potent XKA inhibition of tumor cells is due to specific degradation of the AKT protein and low cellular p53 levels. These finding 
will aid to the development of AKT inhibitors as antitumor agents.

\section{Acknowledgements}

Not applicable.

\section{Funding}

The present study was supported by grants from the Natural Scientific Foundation of China (nos. 81373308 and 31471150) and the Jiangxi Provincial Department of Science and Technology (grant no. 2016BAB204165).

\section{Availability of data and materials}

The datasets used during the present study are available from the corresponding author upon reasonable request.

\section{Authors' contributions}

$\mathrm{QH}$ and CS conceived and designed the study. CC, ZJ, XO and $\mathrm{HZ}$ performed the experiments. $\mathrm{QH}, \mathrm{CC}$ and $\mathrm{CS}$ wrote the paper. $\mathrm{QH}$ and $\mathrm{CS}$ reviewed and edited the manuscript. All authors read and approved the manuscript and agree to be accountable for all aspects of the research in ensuring that the accuracy or integrity of any part of the work are appropriately investigated and resolved.

\section{Ethics approval and consent to participate}

All experimental protocols were approved by the Institutional Review Board.

\section{Consent for publication}

Not applicable.

\section{Competing interests}

The authors declare that they have no competing interests.

\section{References}

1. Manning BD and Toker A: AKT/PKB signaling: Navigating the Network. Cell 169: 381-405, 2017.

2. Mundi PS, Sachdev J, McCourt C and Kalinsky K: AKT in cancer: New molecular insights and advances in drug development. Br J Clin Pharmacol 82: 943-956, 2016.
3. Chan CH, Jo U, Kohrman A, Rezaeian AH, Chou PC, Logothetis $\mathrm{C}$ and Lin HK: Posttranslational regulation of Akt in human cancer. Cell Biosci 4: 59, 2014.

4. Kim D, Dan HC, Park S, Yang L, Liu Q, Kaneko S, Ning J, He L, Yang H, Sun M, et al: AKT/PKB signaling mechanisms in cancer and chemoresistance. Front Biosci 10: 975-987, 2005.

5. Nitulescu GM, Margina D, Juzenas P, Peng Q, Olaru OT, Saloustros E, Fenga C, Spandidos DA, Libra M and Tsatsakis AM: Akt inhibitors in cancer treatment: The long journey from drug discovery to clinical use (Review). Int J Oncol 48: 869-885, 2016.

6. Kumar CC and Madison V: AKT crystal structure and AKT-specific inhibitors. Oncogene 24: 7493-7501, 2005.

7. Jiang ZK, Guo L, Chen C, Liu SW, Zhang L, Dai SJ, He QY, You XF, Hu XX, Tuo L, et al: Xiakemycin A, a novel pyranonaphthoquinone antibiotic, produced by the Streptomyces sp. CC8-201 from the soil of a karst cave. J Antibiot (Tokyo) 68: 771-774, 2015.

8. Sperry J, Bachu P and Brimble MA: Pyranonaphthoquinones - isolation, biological activity and synthesis. Nat Prod Rep 25: 376-400, 2008.

9. Brimble MA, Duncalf LJ and Nairn MR: Pyranonaphthoquinone antibiotics - isolation, structure and biological activity. Nat Prod Rep 16: 267-281, 1999.

10. Jiménez-Alonso S, Orellana HC, Estévez-Braun A, Ravelo AG, Pérez-Sacau E and Machín F: Design and synthesis of a novel series of pyranonaphthoquinones as topoisomerase II catalytic inhibitors. J Med Chem 51: 6761-6772, 2008.

11. Toral-Barza L, Zhang WG, Huang X, McDonald LA, Salaski EJ, Barbieri LR, Ding WD, Krishnamurthy G, Hu YB, Lucas J, et al: Discovery of lactoquinomycin and related pyranonaphthoquinones as potent and allosteric inhibitors of AKT/PKB: Mechanistic involvement of AKT catalytic activation loop cysteines. Mol Cancer Ther 6: 3028-3038, 2007.

12. Korwar S, Nguyen T and Ellis KC: Preparation and evaluation of deconstruction analogues of 7-deoxykalafungin as AKT kinase inhibitors. Bioorg Med Chem Lett 24: 271-274, 2014.

13. Chen J, Chen Y and He Q: Action of bleomycin is affected by bleomycin hydrolase but not by caveolin-1. Int J Oncol 41: 2245-2252, 2012.

14. Chen Y, Zhang H and He Q: Involvement of bleomycin hydrolase and poly(ADP-ribose) polymerase-1 in Ubc9-mediated resistance to chemotherapy agents. Int J Oncol 50: 223-231, 2017.

15. Salaski EJ, Krishnamurthy G, Ding WD, Yu K, Insaf SS, Eid C, Shim J, Levin JI, Tabei K, Toral-Barza L, et al: Pyranonaphthoquinone lactones: A new class of AKT selective kinase inhibitors alkylate a regulatory loop cysteine. J Med Chem 52: 2181-2184, 2009.

16. Léo PM, Morin C and Philouze C: Structure revision of medermycin/lactoquinomycin a and of related C-8 glycosylated naphthoquinones. Org Lett 4: 2711-2714, 2002.

17. Gottlieb TM, Leal JF, Seger R, Taya Y and Oren M: Cross-talk between Akt, p53 and Mdm2: Possible implications for the regulation of apoptosis. Oncogene 21: 1299-1303, 2002.

18. Mayo LD, Dixon JE, Durden DL, Tonks NK and Donner DB: PTEN protects $\mathrm{p} 53$ from $\mathrm{Mdm} 2$ and sensitizes cancer cells to chemotherapy. J Biol Chem 277: 5484-5489, 2002.

19. Sangai T, Akcakanat A, Chen H, Tarco E, Wu Y, Do KA Miller TW, Arteaga CL, Mills GB, Gonzalez-Angulo AM, et al: Biomarkers of response to Akt inhibitor MK-2206 in breast cancer. Clin Cancer Res 18: 5816-5828, 2012.

20. Weinlich R, Oberst A, Beere HM and Green DR: Necroptosis in development, inflammation and disease. Nat Rev Mol Cell Biol 18: 127-136, 2017.

21. Csizmar CM, Kim DH and Sachs Z: The role of the proteasome in AML. Blood Cancer J 6: e503, 2016. 